\title{
Renormalization of Yukawa model with sterile scalar in curved spacetime
}

\author{
Vítor Fernandes Barra $^{1, a}$, Iosif L. Buchbinder ${ }^{2,3, b}$, Jarme Gomes Joaquim ${ }^{1, c}$, Andreza Rairis Rodrigues ${ }^{1, \mathrm{~d}}$, \\ Ilya L. Shapiro ${ }^{1,2,3, \mathrm{e}}$ \\ ${ }^{1}$ Departamento de Física, ICE, Universidade Federal de Juiz de Fora, Juiz de Fora, MG 36036-330, Brazil \\ ${ }^{2}$ Department of Theoretical Physics, Tomsk State Pedagogical University, 634061 Tomsk, Russia \\ ${ }^{3}$ National Research Tomsk State University, 634050 Tomsk, Russia
}

Received: 2 April 2019 / Accepted: 2 May 2019 / Published online: 30 May 2019

(c) The Author(s) 2019

\begin{abstract}
We explore the classical and quantum properties of a sterile scalar field coupled to $N$ copies of Dirac fermions in an external gravitational field. We find that the self-interaction scalar potential of a model that is consistent at the quantum level, includes odd (first and third) powers of a scalar. In particular, one has to consider, besides the standard non-minimal coupling of the form $\xi \varphi^{2} R$, the new type of non-minimal coupling of the form $f \varphi R$ with new non-minimal parameter $f$. We study the one-loop renormalization of such a theory including renormalization of the new non-minimal coupling. Also, we calculate the one-loop effective potential using the renormalization group and show how the renormalization group analysis should be extended compared to the standard expression which was derived in 1980ies. This conclusion is supported by the direct calculation of effective potential using normal coordinates and covariant cut-off regularization. The important features of the classical theory with a sterile scalar are related to the presence of the qualitatively new terms in the induced action of gravity, coming from the odd terms. We show that this new feature of the theory may have phenomenologically relevant consequences, both in the low-energy gravitational physics and at the high energies, corresponding to inflation.
\end{abstract}

\footnotetext{
a e-mail: vitorbarra@ice.ufjf.br

b e-mail: joseph@tspu.edu.ru

c e-mail: jarme@ice.ufjf.br

d e-mail: andrezarodrigues@ice.ufjf.br

e e-mails: ilyashapiro2003@yahoo.com.br; ilyashapiro2016@gmail.com
}

\section{Introduction}

The unique scalar field of the Minimal Standard Model (MSM) of particle physics is the Higgs boson, which is complex and belongs to the fundamental representation of $S U(2)$. The extensions of MSM such as nonminimal, supersymmetric version, of Grand Unification Theories, typically have larger scalar sector, but the new scalars are always representations of the symmetry group of the corresponding particle physics model. The consistency of such models with respect to quantum field theory requirements are the main tool in restricting the extensions of MSM, and in particular the scalar sector.

At the same time there is another sort of scalar fields, which are intensively used in cosmology. Both inflaton and quintessence are real scalars which are not related to representations of the symmetry group of particle physics and which can be called sterile scalars. An interesting question concerns the restrictions which can be imposed in quantum theory on the self-interaction potential of such a scalar field. A practical realization of this program requires assuming the form of the interaction between the sterile scalar and elementary particles. In the present work we consider the simplest possible version of such interaction, that means we consider a sterile scalar coupled to the $N$ copies of massive Dirac fermion through Yukawa interaction.

It is well known that the multiplicative renormalization of a scalar field in curved space-time requires introducing the non-minimal coupling between scalar field and gravity in the form $\xi \varphi^{2} R$. However, if the classical potential of the scalar field includes $\varphi^{3}$-term, one can expect that the renormalized 
theory should include the new type of the non-minimal coupling proportional to $\varphi R$, with the new nonminimal parameter. Thus, we arrive at the problem of describing the quantum structure of the theory with a sterile scalar. The purpose of this paper is to consider the main aspects of this problem, such as renormalization and renormalization group. As far as we know, these subjects have not been studied in the literature, so far.

To study the above new aspects we start by using the standard heat kernel approach to derive the one-loop divergences in such a model in curved space-time. As a result of this calculation we arrive at the minimal form of consistent selfinteracting potential which provides multiplicative renormalizability of the model. The main new feature of this potential in comparison with a usual scalar (e.g. Higgs) is the presence of third and first powers of scalar field in the classical potential. Let us note that in the previous existing calculations of similar divergences in Refs. [1-4] the presence of these terms was recognized, but the consequences of this aspect of the theory were never sufficiently well explored. We fill this gap in the present work, including the discussion of the role of odd terms for the induced action of gravity, when the scalar field is in the minimum of the potential of the sterile scalar. Another interesting aspect of the sterile scalar coupled to fermions through the Yukawa interaction is the possible role of the odd terms is inflation, that will be also addressed in what follows.

From the formal QFT side, the challenging problem is how to take into account the renormalization group for the odd terms, especially when it concerns the renormalization group-based derivation of the effective potential. The standard expression for effective potential restored from the renormalization group equation for effective action in curved space [5] (see also further development of the renormalization group method, applied for other sectors of effective action in [6]) is valid only for the theory where divergences have only second and fourth powers of the scalar field. The effective potential in the model with Yukawa interaction was calculated recently in $[3,4]$ for the special case of massless fermions, when the odd terms in the scalar potential are not necessary and the loop contributions to the potential can be derived on the basis of the standard general expressions in flat [7] and curved spacetime [2,5]. If fermions are massive and there are odd terms, these standard results are incomplete and the renormalization group derivation of the effective potential should be modified somehow. As usual (see the discussion in [8]), the renormalization group derivation is based on the identification of the renormalization parameter $\mu$, which enables one to easily go beyond the local potential approximation $[2,6]$. On the other hand, such identification represents an assumption, which is always good to check, at least in the simplest case of effective potential. Thus, in order to achieve an additional verification of the complete result, we perform the derivation of effective potential for a sterile scalar directly, using the method which was developed recently in [8] in the basis of Riemann normal coordinates and local momentum representation $[9,10]$.

The paper is organized as follows. In the next Sect. 2 we describe the derivation of one-loop divergences in the model with a sterile scalar coupled to $N$-component fermion. The beta- and gamma-functions are calculated, and the one-loop effective potential restored from the renormalization group in Sect. 3 . In Sect. 4 we present the tree-level analysis of the curved-space analog of the spontaneous symmetry breaking, in the presence of odd scalar terms, using an approximation of small odd terms and weak gravitational field. The analysis of the induced gravitational action in Sect. 5 shows that in the presence of odd terms, for a light scalar case, there are unusual low-energy terms, which can be phenomenologically interesting. At another end of energy spectrum we meet small but potentially detectable effects of the odd terms. Depending on the details of the particle physics model at high energies, these terms may be within the reach of the possible observations. Finally, in Sect. 6 we draw our conclusions and discuss the perspectives for further work.

\section{Yukawa model with sterile scalar and one-loop divergences}

Consider Yukawa model with a single real sterile scalar coupled to the $N$ copies of fermion field. It proves useful to choose the classical action of the form

$$
\begin{aligned}
S= & \int d^{4} x \sqrt{-g}\left\{i \bar{\Psi}_{i}\left(\gamma^{\mu} \nabla_{\mu}+i M+i h \varphi\right) \delta^{i j} \Psi_{j}\right. \\
& +\frac{1}{2}\left(g^{\mu \nu} \partial_{\mu} \varphi \partial_{\nu} \varphi-m^{2} \varphi^{2}+\xi R \varphi^{2}\right)-\frac{\lambda}{4 !} \varphi^{4} \\
& \left.-\frac{g}{3 !} \varphi^{3}-\tau \varphi-f R \varphi\right\},
\end{aligned}
$$

where $m$ is a scalar fields mass, $M$ is a spinor field mass, $h$ is the Yukawa coupling constant, $\lambda, g$ and $\tau$ are the coupling constants in the scalar sector which survive in the flat limit, while $\xi$ and $f$ are the non-minimal parameters of scalar field coupling to gravity. The terms with odd powers and correspondingly the parameters $g, \tau$ and $f$ were not analysed in detail in the previous considerations of the model in [1-4], 
regardless the odd terms were identified in the one-loop divergences. From the formal quantum field theory point of view, the consistent theory of a sterile scalar coupled to fermions should include these terms from the very beginning and this is the approach we are starting to pursue in this work.

Regardless of the calculation of one-loop divergences in this theory follows the standard procedure (see, e.g., [2] for a number of well-elaborated examples), we shall give some details below, in order to simplify possible verifications. Let us stress from the very beginning that gravity will not be quantized in this paper (indeed, the generalization to the case of quantum gravity can be found for similar models in the original papers [11-13] and book [2], where the one-loop calculations were done by means of the generalized SchwingerdeWitt technique, and the subsequent recent work [14], where similar calculations were performed by using Feynman diagrams (without much detail) and used for attempting to reconciliate the renormalizability of quantum gravity and the absence of higher derivative ghosts.

Let us start by decomposing the matter fields into classical $\varphi, \bar{\Psi}, \Psi$ and quantum $\sigma, \bar{\eta}, \eta$ counterparts,

$\varphi \rightarrow \varphi+\sigma, \quad \bar{\Psi}_{i} \rightarrow \bar{\Psi}_{i}+\bar{\eta}_{i}$,

$$
\Psi_{j} \rightarrow \Psi_{j}+\eta_{j}
$$

The one-loop divergences are defined by the bilinear part of the action, which involves the operator $\hat{H}$,

$$
\begin{aligned}
S^{(2)}= & \frac{1}{2} \int d^{4} x \sqrt{-g}\left(\begin{array}{ll}
\sigma & \bar{\eta}_{i}
\end{array}\right) \hat{H}\left(\begin{array}{c}
\sigma \\
\eta_{j}
\end{array}\right) \\
= & \frac{1}{2} \int d^{4} x \sqrt{-g}\left\{\sigma H_{11} \sigma+\bar{\eta}_{i} H_{21} \sigma\right. \\
& \left.+\sigma H_{12} \eta_{j}+\bar{\eta}_{i} H_{22} \eta_{j}\right\},
\end{aligned}
$$

or in the explicit form

$$
\begin{aligned}
S^{(2)}= & \frac{1}{2} \int d^{4} x \sqrt{-g}\left\{2 i \bar{\eta}_{i}(\not \nabla+i M) \eta_{j} \delta^{i j}-\sigma \square \sigma\right. \\
& -m^{2} \sigma^{2}+\xi R \sigma^{2}-2 h\left(\varphi \bar{\eta}_{i} \eta_{j}+\sigma \bar{\Psi}_{i} \eta_{j}+\sigma \bar{\eta}_{i} \Psi_{j}\right) \delta^{i j} \\
& \left.-\frac{\lambda}{2} \sigma^{2} \varphi^{2}-g \varphi \sigma^{2}\right\} .
\end{aligned}
$$

Here the quadratic in quantum fields action $S^{(2)}$ depends on the background gravitational field and the background fields $\bar{\Psi}, \Psi, \varphi$. After some algebra we get

$$
\hat{H}=\left(\begin{array}{cc}
\xi R-\square-m^{2}-g \varphi-\frac{\lambda}{2} \varphi^{2} & -2 h \bar{\Psi}_{j} \\
-2 h \Psi_{i} & 2 i(\not \nabla+i M+i h \varphi) \delta^{i j}
\end{array}\right) .
$$

In order to reduce the problem of deriving $\ln$ Det $\hat{H}$ to the standard form, one can introduce the conjugated matrix oper- ator $\hat{H}^{*}$ as follows,

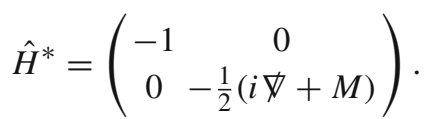

It is well-known that the one-loop effective action has the form $\sim \operatorname{Tr} \ln (\hat{H})$. To calculate the divergences of effective action we will write it as

$\operatorname{Tr} \ln (\hat{H})=\operatorname{Tr} \ln \left(\hat{H} \hat{H}^{*}\right)-\operatorname{Tr} \ln \left(\hat{H}^{*}\right)$.

It is easy to see that $\operatorname{Tr} \ln \hat{H}^{*}$ contributes only to the vacuum divergences, that are already known for an arbitrary model $[2,15]$. Therefore it is sufficient to calculate the divergences of the product $\hat{H} \hat{H}^{*}$, which has a standard form,

$\hat{\mathcal{H}}=\hat{H} \hat{H}^{*}=\hat{1} \square+2 \hat{h}^{\mu} \nabla_{\mu}+\hat{\Pi}$,

where we can identify

$\hat{h}^{\mu}=\left(\begin{array}{cc}0 & \frac{i}{2} h \bar{\Psi}_{j} \gamma^{\mu} \\ 0 & \frac{i}{2} h \varphi \gamma^{\mu} \delta^{i j}\end{array}\right)$

and

$$
\hat{\Pi}=\left(\begin{array}{cc}
m^{2}+\frac{\lambda}{2} \varphi^{2}-\xi R+g \varphi & h M \bar{\Psi}_{j} \\
2 h \Psi_{i} & \delta^{i j}\left[M^{2}-\frac{1}{4} R+h M \varphi\right]
\end{array}\right) .
$$

The one-loop divergences can be derived by means of the Schwinger-De-Witt technique (see, e.g. [2,16]) and are given by the general expression

$$
\begin{aligned}
\Gamma_{d i v}^{(1)}= & -\frac{\mu^{D-4}}{\varepsilon} \int d^{D} x \sqrt{-g} \operatorname{Tr}\left\{\frac{1}{2} \hat{P}^{2}+\frac{1}{12} \hat{S}_{\mu \nu}^{2}\right. \\
& \left.+\frac{1}{6} \square \hat{P}+\frac{\hat{1}}{180}\left(R_{\mu \nu \alpha \beta}^{2}-R_{\mu \nu}^{2}+\square R\right)\right\},
\end{aligned}
$$

where $\varepsilon=(4 \pi)^{2}(D-4)$ and

$$
\begin{aligned}
\hat{P}= & \hat{\Pi}+\frac{\hat{1}}{6} R-\nabla_{\mu} \hat{h}^{\mu}-\hat{h}_{\mu} \hat{h}^{\mu}, \\
\hat{S}_{\mu \nu}= & {\left[\nabla_{\nu}, \nabla_{\mu}\right] \hat{1}+\nabla_{\nu} \hat{h}_{\mu}-\nabla_{\mu} \hat{h}_{\nu} } \\
& +\hat{h}_{\nu} \hat{h}_{\mu}-\hat{h}_{\mu} \hat{h}_{\nu} .
\end{aligned}
$$

We give intermediate formulas in the Appendix, and here present only the final result,

$$
\begin{aligned}
\Gamma_{d i v}^{(1)}= & -\frac{\mu^{D-4}}{\varepsilon} \int d^{D} x \sqrt{-g}\left\{\frac{m^{4}}{2}-2 N M^{4}\right. \\
& +\left[\frac{N}{3} M^{2}-m^{2}\left(\xi-\frac{1}{6}\right)\right] R \\
& +\left(\frac{N}{24}+\frac{1}{45}\right) R_{\mu \nu \alpha \beta}^{2}+\frac{8 N-1}{180} R_{\mu \nu}^{2} \\
& -\left[\frac{1}{2}\left(\xi-\frac{1}{6}\right)^{2}+\frac{N}{18}\right] R^{2}
\end{aligned}
$$




$$
\begin{aligned}
& +\left[\frac{5 N+1}{45}-\frac{1}{6}\left(\xi-\frac{1}{6}\right)\right] \square R \\
& +\sum_{k} 3 i h^{2} \bar{\Psi}_{k}\left[\frac{1}{2} \not \nabla-i M-i h \varphi\right] \Psi_{k}+2 N h^{2}\left(\partial_{\mu} \varphi\right)^{2} \\
& +\frac{1}{6}(g \varphi-8 N h M) \square \varphi+\frac{1}{12}\left(\lambda-16 N h^{2}\right) \square \varphi^{2} \\
& -\frac{1}{2}\left[\left(\xi-\frac{1}{6}\right) \lambda-\frac{2}{3} N h^{2}\right] R \varphi^{2}-\left[g\left(\xi-\frac{1}{6}\right)-\frac{2}{3} N h M\right] R \varphi \\
& +\left(m^{2} g-8 N h M^{3}\right) \varphi+\frac{1}{2}\left(g^{2}+\lambda m^{2}-24 N h^{2} M^{2}\right) \varphi^{2} \\
& \left.+\left(\frac{1}{8} \lambda^{2}-2 N h^{4}\right) \varphi^{4}-\left(8 N M h^{3}-\frac{1}{2} g \lambda\right) \varphi^{3}\right\},
\end{aligned}
$$

where the vacuum divergences were also included for completeness.

A few general comments are in order at this point. First of all, the result (13) confirms our expectations. All odd terms which we included into the classical action (3) really emerge in the one-loop divergences. The reason is that in the theory with sterile scalar these terms are not protected by any kind of symmetry, and hence it was actually expected that they would show up. Second, as far as we have the odd-power divergences, one should expect the logarithmic contributions in the corresponding finite part of effective action, in particular in the effective potential of a sterile scalar. In the next sections we shall see that these expectation will be completely confirmed. Third, it is worth pointing out that the odd terms may affect on the form of the non-local form factors, similar to what we had in the Yukawa model for the even terms [17] and earlier for a self-interacting scalar [18]. The discussion of this issue goes beyond the framework of the present work and will be left for the future.

The renormalization relations between bare and renormalizable quantities have the form which directly follows from the divergences. For the fields we have

$$
\begin{aligned}
\varphi_{0} & =\mu^{\frac{D-4}{2}}\left(1+\frac{2 N h^{2}}{\epsilon}\right) \varphi, \\
\Psi_{k 0} & =\mu^{\frac{D-4}{2}}\left(1+\frac{3}{4 \epsilon} h^{2}\right) \Psi_{k}, \\
\bar{\Psi}_{k 0} & =\mu^{\frac{D-4}{2}}\left(1+\frac{3}{4 \epsilon} h^{2}\right) \bar{\Psi}_{k} .
\end{aligned}
$$

The relations for masses have the form

$$
\begin{aligned}
& M_{0}=\left(1-\frac{9}{2 \epsilon} h^{2}\right) M, \\
& m_{0}^{2}=m^{2}-\frac{g^{2}+4 N h^{2} m^{2}+\lambda m^{2}-24 N h^{2} M^{2}}{\epsilon} .
\end{aligned}
$$

For the usual even couplings and nonminimal parameters we have

$$
\begin{aligned}
& \xi_{0}=\xi-\frac{\lambda+4 N h^{2}}{\epsilon}\left(\xi-\frac{1}{6}\right), \\
& h_{0}=\mu^{\frac{4-D}{2}} h\left(1-\frac{4 N h^{2}+9 h^{2}}{2 \epsilon}\right), \\
& \lambda_{0}=\mu^{4-D}\left(\lambda+\frac{48 N h^{4}-8 N \lambda h^{2}-3 \lambda^{2}}{\epsilon}\right) .
\end{aligned}
$$

And, finally, for the odd couplings and nonminimal parameters,

$$
\begin{aligned}
g_{0} & =\mu^{\frac{4-D}{2}}\left(g+\frac{48 N M h^{3}-3 g \lambda-6 N h^{2} g}{\epsilon}\right), \\
\tau_{0} & =\mu^{\frac{D-4}{2}}\left(\tau+\frac{8 N h M^{3}-2 N \tau h^{2}-m^{2} g}{\epsilon}\right), \\
f_{0} & =\mu^{\frac{D-4}{2}}\left[f+\frac{g}{\epsilon}\left(\xi-\frac{1}{6}\right)-\frac{2 N h M+6 N f h^{2}}{3 \epsilon}\right] .
\end{aligned}
$$

These expressions demonstrate the non-trivial renormalization of the odd coupling parameters, including the new nonminimal parameter $f$.

\section{Renormalization group and effective potential}

In this section we consider the renormalization group equation for the effective potential and discuss its solution to derive the effective potential for the model under consideration up to first order in scalar curvature. The form of the equation is defined by the corresponding beta-and gammafunctions which are calculated on the basis of the renormalization relations for the parameters and fields (for the theories in curved space time see e.g. [2, 19,20]).

Let us begin with beta-functions . They are defined as follows

$\beta_{P}=\lim _{D \rightarrow 4} \mu \frac{d P}{d \mu}$,

where $P=\{m, M, h, \lambda, \xi, g, \tau, f\}$ are the renormalized parameters. The scheme of derivation is described in [2] and we will not repeat it here, but only present the results. The calculation which is based on the relations (18), (21), (24) leads to

$$
\begin{aligned}
\beta_{h} & =\frac{(4 N+9) h^{3}}{(4 \pi)^{2}}, \\
\beta_{M} & =\frac{9 h^{2} M}{2(4 \pi)^{2}}, \\
\beta_{\lambda} & =\frac{1}{(4 \pi)^{2}}\left(8 N \lambda h^{2}+3 \lambda^{2}-48 N h^{4}\right),
\end{aligned}
$$




$$
\begin{aligned}
\beta_{\xi} & =\frac{1}{(4 \pi)^{2}}\left(4 N h^{2}+\lambda\right)\left(\xi-\frac{1}{6}\right), \\
\beta_{g} & =\frac{1}{(4 \pi)^{2}}\left(\frac{3}{2} g \lambda+3 N g h^{2}-12 N M h^{3}\right), \\
\beta_{m^{2}} & =\frac{1}{(4 \pi)^{2}}\left[m^{2} \lambda+g^{2}+\left(4 m^{2}-24 M^{2}\right) N h^{2}\right], \\
\beta_{\tau} & =\frac{1}{(4 \pi)^{2}}\left(2 N \tau h^{2}+g m^{2}-8 N h M^{3}\right), \\
\beta_{f} & =\frac{1}{(4 \pi)^{2}}\left[2 N f h^{2}-g\left(\xi-\frac{1}{6}\right)+\frac{2}{3} N M h\right] .
\end{aligned}
$$

The gamma-functions are defined as follows:

$$
\lim _{D \rightarrow 4} \mu \frac{d \Phi}{d \mu}=\gamma_{\Phi} \Phi
$$

where $\Phi$ are the renormalized fields, $\Phi=\left(\varphi, \Psi_{k}\right)$. The relations (16) lead to

$$
\begin{aligned}
\gamma_{\varphi} & =-\frac{2 N h^{2}}{(4 \pi)^{2}}, \\
\gamma_{\Psi_{k}} & =-\frac{3 h^{2}}{4(4 \pi)^{2}} .
\end{aligned}
$$

In the case of conformal invariant theory we should put all dimensional constants $m^{2}, M, g, \tau$ and $f$ vanish and set $\xi=\frac{1}{6}$. It is easy to see that in this situation the pole coefficient in the expression for the divergences (13) is also conformal invariant. Furthermore, the beta functions have the corresponding conformal fixed point, as it has to be from the general perspective [2,19].

Now we briefly discuss how to find the one-loop effective potential from the $\overline{\mathrm{MS}}$ renormalization group equation in curved spacetime. The starting point is the overall $\mu$ independence of effective action,

$\mu \frac{d}{d \mu} \Gamma\left[g_{\alpha \beta}, \Phi, P, n, \mu\right]=0$,

which immediately leads to [2,19]

$$
\begin{aligned}
& \left\{\mu \frac{\partial}{\partial \mu}+\beta_{P} \frac{\partial}{\partial P}+\int d^{D} x \gamma_{\Phi} \Phi \frac{\delta}{\delta \Phi(x)}\right\} \\
& \quad \times \Gamma\left[g_{\alpha \beta}, \Phi, P, D, \mu\right]=0,
\end{aligned}
$$

where we assume the sum over all parameters $P$ and the fields $\Phi=\left(\varphi, \Psi_{k}\right)$. From now on we shall set $D=4$.

The effective potential is defined as zero-order approximation in the derivative expansion for the scalar sector of $\Gamma$,

$$
\begin{aligned}
\Gamma\left[g_{\alpha \beta}, \varphi, P, \mu\right]= & \int d^{4} x \sqrt{-g}\left\{-V_{e f f}\left(\varphi, g_{\alpha \beta}\right)\right. \\
& \left.+\frac{1}{2} Z\left(\varphi, g_{\alpha \beta}\right) g^{\mu \nu} \partial_{\mu} \varphi \partial_{\nu} \varphi+\cdots\right\} .
\end{aligned}
$$

Since (31) is a linear homogeneous equation, we get

$$
\left\{\mu \frac{\partial}{\partial \mu}+\beta_{P} \frac{\partial}{\partial P}+\gamma_{\varphi} \varphi \frac{\partial}{\partial \varphi}\right\} V_{e f f}\left(g_{\alpha \beta}, \varphi, P, \mu\right)=0 .
$$

Equation (33) means that the explicit functional dependence on $\mu$ in the effective potential is exactly compensated by the $\mu$-dependence of the scalar field $\varphi$ and parameters $P$. At the one-loop level the last dependence can be written in the simple form involving the first order logarithmic dependence, as one can figure out from the above expressions for beta- and gamma-functions.

We will search for the effective potential up to the terms linear in scalar curvature, $V_{\text {eff }}=V_{0}+R V_{1}$, where $V_{0}$ is the flat-space effective potential and $R V_{1}$ is the first curvaturedependent correction to $V_{0}$. Both functions $V_{0}$ and $V_{1}$ satisfy Eq. (33). Before solving the equations for $V_{0}$ and $V_{1}$, we will take into account that in the one-loop approximation each spin gives additive contribution to the effective action. Therefore we can write $V_{0}=V_{0}^{(0)}+V_{0}^{\left(\frac{1}{2}\right)}$ and $V_{1}=V_{1}^{(0)}+$ $V_{1}^{\left(\frac{1}{2}\right)}$, where the labels $(0)$ and $\left(\frac{1}{2}\right)$ mean contribution from quantum scalar and spinor fields, respectively. The quantities $V_{0}^{(0)}$ and $V_{0}^{\left(\frac{1}{2}\right)}$ correspond to the theory under consideration in flat space. In this section we demonstrate how they can be found starting from the renormalization group equation.

Let us begin with finding $V_{0}^{(0)}$. The equation for this quantity has the form

$$
\left\{\mu \frac{\partial}{\partial \mu}+\beta_{P} \frac{\partial}{\partial P}+\gamma_{\varphi} \varphi \frac{\partial}{\partial \varphi}\right\} V_{0}^{(0)}(\varphi, P, \mu)=0 .
$$

The Eq. (34) is a complicated partial differential equation with non-constant coefficients. Before solving this equation, it proves helpful to bring qualitative considerations that simplify the solution. The parameter $\mu$ can enter to the solution for $V_{0}^{(0)}$ only logarithmically. Since the argument of the logarithm must be dimensionless, the dependence on $\mu$ should be through the parameter $t=\frac{1}{2} \ln \frac{X}{\mu^{2}}$, where the quantity $X$ has the mass dimension two. This quantity can be constructed only from the dimensional parameters of the classical action, i.e. from $m, M, \varphi, g, \tau$ with arbitrary dimensionless coefficients. In principle, these coefficients should be fixed with the help of appropriate renormalization conditions for the effective potential. However, it is natural to assume that the form of effective potential should be consistent with the form of the operator $\hat{\mathcal{H}}(8)$ in the scalar sector. It means that the most natural choice for $X$ is $X^{(0)}=m^{2}+g \varphi+\frac{1}{2} \lambda \varphi^{2}$. Thus, we identify 
$t^{(0)}=\frac{1}{2} \ln \frac{m^{2}+\frac{1}{2} \lambda \varphi^{2}+g \varphi}{\mu^{2}}$

for the scalar field contribution to effective potential. The parameters $M$ and $h$ do not contribute in the scalar sector. Therefore $V_{0}^{(0)}=V_{0}^{(0)}\left(t, m^{2}, g, \tau, \varphi\right)$ and the Eq. (34) becomes

$$
\begin{gathered}
\left\{\mu \frac{\partial}{\partial \mu}+\beta_{m^{2}}^{(0)} \frac{\partial}{\partial m^{2}}+\beta_{\lambda}^{(0)} \frac{\partial}{\partial \lambda}+\beta_{g}^{(0)} \frac{\partial}{\partial g}\right. \\
\left.+\beta_{\tau}^{(0)} \frac{\partial}{\partial \tau}+\gamma_{\varphi}^{(0)} \varphi \frac{\partial}{\partial \varphi}\right\} V_{0}^{(0)}=0 .
\end{gathered}
$$

Here the functions $\beta_{\lambda}^{(0)}, \beta_{g}^{(0)}, \beta_{m^{2}}, \gamma_{\varphi}^{(0)}$ and $\beta_{\tau}^{(0)}$ are taken at $M=0$ and $h=0$. For the scalar field contribution $\gamma_{\varphi}^{(0)}=$ 0 . The next step is to express the derivative with respect to $\mu$ through the derivative with respect to the parameter $t^{(0)}$ defined in (35). As a result, the Eq. (36) looks like

$$
\begin{gathered}
\left\{\frac{\partial}{\partial t^{(0)}}-\bar{\beta}_{m^{2}}^{(0)} \frac{\partial}{\partial m^{2}}-\bar{\beta}_{\lambda}^{(0)} \frac{\partial}{\partial \lambda}-\bar{\beta}_{g}^{(0)} \frac{\partial}{\partial g}\right. \\
\left.-\bar{\beta}_{\tau}^{(0)} \frac{\partial}{\partial \tau}-\bar{\gamma}_{\varphi}^{(0)} \varphi \frac{\partial}{\partial \varphi}\right\} V_{0}^{(0)}=0,
\end{gathered}
$$

where

$$
\begin{aligned}
\left(\bar{\beta}_{m^{2}}^{(0)},\right. & \left.\bar{\beta}_{\lambda}^{(0)}, \bar{\beta}_{g}^{(0)}, \bar{\beta}_{\tau}^{(0)}, \bar{\gamma}_{\varphi}^{(0)}\right) \\
& =\frac{1}{1-Q^{(0)}}\left(\beta_{m^{2}}^{(0)}, \beta_{\lambda}^{(0)}, \beta_{g}^{(0)}, \beta_{\tau}^{(0)}, \gamma_{\varphi}^{(0)}\right)
\end{aligned}
$$

and

$Q^{(0)}=1-\frac{\partial t^{(0)}}{\partial m^{2}}-\frac{\partial t^{(0)}}{\partial \lambda}-\frac{\partial t^{(0)}}{\partial \varphi}-\frac{\partial t^{(0)}}{\partial g}$.

Solution to the Eq. (37) is written as follows

$$
\begin{aligned}
& V_{0}^{(0)}\left(t^{(0)}, m^{2}, \lambda, g, \tau, \varphi\right) \\
& =V_{0} c l\left(m^{2}\left(t^{(0)}\right), \lambda\left(t^{(0)}\right), g\left(t^{(0)}\right), \tau\left(t^{(0)}\right), \varphi\left(t^{(0)}\right),\right.
\end{aligned}
$$

where

$V_{0 c l}=\frac{1}{2} m^{2} \varphi^{2}+\frac{\lambda}{4 !} \varphi^{4}+\frac{g}{3 !} \varphi^{3}+\tau \varphi$

is the classical potential and $m^{2}\left(t^{(0)}\right), \lambda\left(t^{(0)}\right), g\left(t^{(0)}\right), \tau\left(t^{(0)}\right)$ and $\varphi\left(t^{(0)}\right)$ are the running parameters $P\left(t^{(0)}\right)$ and the scalar field, satisfying the equations

$$
\begin{aligned}
& \frac{d P\left(t^{(0)}\right)}{d t^{(0)}}=\bar{\beta}_{P}^{(0)}\left(t^{(0)}\right), \\
& \frac{d \varphi\left(t^{(0)}\right)}{d t^{(0)}}=\bar{\gamma}_{\varphi}\left(t^{(0)}\right)
\end{aligned}
$$

with the initial conditions

$$
P(t)_{\mid t=0}=P .
$$

As before, here $P=m^{2}, \lambda, g, \tau$. Since we work in the one-loop approximation, all quantum corrections are linear in $\hbar$, hence we can set the quantity $Q$ (39) equal to zero in the expressions for beta- and gamma-functions. Then the solutions of the Eq. (42) can be easily found

$$
\begin{aligned}
& P\left(t^{(0)}\right)=P+\beta_{P}^{(0)} t^{(0)}, \\
& \varphi\left(t^{(0)}\right)=\varphi+\gamma_{\varphi}^{(0)} t^{(0)}=\varphi,
\end{aligned}
$$

where we took into account that $\gamma^{(0)}=0$. The relations (44) together with the explicit forms for the functions $\beta_{P}^{(0)}$ represent the solution for the effective potential $V_{0}^{(0)}$.

The analysis of the $V_{1}^{(0)}$ can be done in a similar way, so we skip the details. The result has the following form

$V_{1}^{(0)}=V_{1 c l}\left(P_{1}\left(t^{(0)}\right), \varphi\left(t^{(0)}\right)\right)$

with $V_{1 c l} R=-\frac{1}{2} \xi R \varphi^{2}+f R \varphi$. The quantities $P_{1}\left(t^{(0)}\right)=$ $P_{1}+\beta_{P_{1}}^{(0)} t^{(0)}$ and $\beta_{P_{1}}^{(0)}=\left(\beta_{\xi}^{(0)}, \beta_{f}^{(0)}\right)$. These relations together with (45) are final solutions for curvature dependent contribution to effective potential from quantum scalar field.

We now turn to finding the quantum contribution $\bar{V}_{0}^{\left(\frac{1}{2}\right)}+$ $R \bar{V}_{1}^{\left(\frac{1}{2}\right)}$ to effective potential from quantum spinor field. In this case we begin with Eq. (33) for $V_{0}^{\left(\frac{1}{2}\right)}$ and $V_{1}^{\left(\frac{1}{2}\right)}$ separately taking into account that consistence with form of the operator $\hat{H}$ in fermionic sector (5) motivates a natural choice for dimensionless parameter containing the logarithm of $\mu$ in the form

$t^{\left(\frac{1}{2}\right)}=\frac{1}{2} \ln \frac{(M+h \varphi)^{2}}{\mu^{2}}$.

All other considerations are analogous to one for $V_{0}^{(0)}$ and $V_{1}^{(0)}$. Thus, we present only the final results for quantum corrections,

$\bar{V}_{1}^{\left(\frac{1}{2}\right)}=V_{0 c l}\left(P^{\left(\frac{1}{2}\right)}\left(t^{\left(\frac{1}{2}\right)}\right), \varphi\left(t^{\left(\frac{1}{2}\right)}\right)\right)$,

and

$R \bar{V}_{1}^{\left(\frac{1}{2}\right)}=R V_{1 c l}\left(P_{1}^{\left(\frac{1}{2}\right)}\left(t^{\left(\frac{1}{2}\right)}\right), \varphi\left(t^{\left(\frac{1}{2}\right)}\right)\right)$,

where the running parameters and field have the form

$$
\begin{aligned}
P^{\left(\frac{1}{2}\right)}\left(t^{\left(\frac{1}{2}\right)}\right) & =\beta_{P}^{\left(\frac{1}{2}\right)} t^{\left(\frac{1}{2}\right)}, \\
\varphi\left(t^{\left(\frac{1}{2}\right)}\right) & =\gamma_{\varphi}^{\left(\frac{1}{2}\right)} t^{\left(\frac{1}{2}\right)} .
\end{aligned}
$$

Here we have solved the equations for running parameters and field with zero initial conditions since the classical contribution to effective potential has been already found when we calculated $V_{0}^{(0)}$ and $V_{1}^{(0)}$. The functions $\beta_{P}^{\left(\frac{1}{2}\right)}$ and $\gamma_{\varphi}^{\left(\frac{1}{2}\right)}$ in the relations (49) are the $\beta_{P}$ and $\gamma_{\varphi}$ at non-zero $M$ and $h$ but with zero parameters $m^{2}, \lambda, g, \tau, \xi$. The relations (47) and 
(48) define the final contribution to effective potential from quantum spinor field.

Thus, we are in a position to present an explicit expression for the Minimal Subtraction scheme-based effective potential,

$$
\begin{aligned}
V_{e f f}= & -\frac{1}{2} m^{2} \varphi^{2}-\frac{1}{2} \xi R \varphi^{2}+\frac{\lambda}{4 !} \varphi^{4}+\frac{g}{3 !} \varphi^{3}+\tau \varphi+f R \varphi \\
& -\frac{1}{2(4 \pi)^{2}}\left\{\left[12 N M^{2} h^{2} t^{\left(\frac{1}{2}\right)}+\frac{\lambda m^{2}-g^{2}}{2} t^{(0)}+C_{1}\right] \varphi^{2}\right. \\
& +\left[\frac{\lambda}{2}\left(\xi-\frac{1}{6}\right) t^{(0)}-\frac{N h^{2}}{3} t^{\left(\frac{1}{2}\right)}+C_{2}\right] R \varphi^{2} \\
& -\frac{1}{3 !}\left[\frac{3 \lambda g}{2} t^{(0)}-3 N\left(g h^{2}-4 M h^{3}\right) t^{\left(\frac{1}{2}\right)}+C_{3}\right] \varphi^{3} \\
& -\frac{1}{4 !}\left[3 \lambda^{2} t^{(0)}-48 N h^{4} t^{\left(\frac{1}{2}\right)}+C_{4}\right] \varphi^{4} \\
& +\left[g m^{2} t^{(0)}+8 N h M^{3} t^{\left(\frac{1}{2}\right)}+C_{5}\right] \varphi \\
& \left.+\left[\frac{2 N M h}{3} t^{\left(\frac{1}{2}\right)}-g\left(\xi-\frac{1}{6}\right) t^{(0)}+C_{6}\right] R \varphi\right\}
\end{aligned}
$$

where $t^{(0)}$ and $t^{\left(\frac{1}{2}\right)}$ were identified in (35) and (46). The constants $C_{1} \ldots 6$ can be found from the initial renormalization conditions. For instance, the two well-known values which correspond to the standard choices in the massless scalar case are $C_{4}=-\frac{25}{6}$ obtained in [7] and $C_{2}=-3$ obtained in [5] (see [2] for a more pedagogical derivation). Since this calculation of the values of $C_{1 \ldots 6}$ for the massive theory is rather cumbersome and there are no immediate applications, we skip it. Let us stress that the quantum corrections in Eq. (50) have qualitatively new terms with odd powers of a sterile scalar field, multiplied by the two kinds of logs. The final definition of the corresponding renormalization constants $C_{3,5,6}$ requires independent measurements and can be achieved only within an appropriate experimental or observational framework. Some of the possible observables related to the odd terms will be discussed in Sect. 5 .

\section{Direct calculation of effective potential}

The results of the previous sections have shown the importance of the terms which are odd in the scalar field. This is something we learned from the divergences derived in the framework of the Scwinger-DeWitt method. Due to the importance of this quantum calculation, it looks reasonable to control its output by qualitatively different method. This is done in the present section by deriving effective potential in the $\mathcal{O}(R)$-approximation using the normal coordinates and local momentum representation, in a way similar to what was done recently in [8], where one can find many relevant technical details and further references.

\subsection{Riemann normal coordinates and scalar contribution}

These coordinates are related to the geodesic lines which link a fiducial point $P^{\prime}\left(x^{\mu^{\prime}}\right)$ with another point with the coordinates $x^{\mu}=x^{\prime \mu}+y^{\mu}$. In order to use the local momentum representation we assume that $g_{\mu \nu}\left(P^{\prime}\right)=\eta_{\mu \nu}$. In the vicinity of this point, in the linear in curvature approximation, we have

$g_{\alpha \beta}(x)=\eta_{\alpha \beta}\left(x^{\prime}\right)-\frac{1}{3} R_{\alpha \mu \beta \nu}\left(x^{\prime}\right) y^{\mu} y^{\nu}+\cdots$.

Then, the bilinear operator in the scalar sector can be written as

$$
-\hat{H}=\frac{1}{\sqrt{-g}} \frac{\delta^{2} S_{\text {scalar }}}{\delta \varphi(x) \delta \varphi\left(x^{\prime}\right)}=\square+V^{\prime \prime},
$$

where $V^{\prime \prime}$ is the second derivative of the classical potential

$V(\varphi)=\frac{1}{2} m^{2} \varphi^{2}+\frac{\lambda}{4 !} \varphi^{4}+\frac{g}{3 !} \varphi^{3}+\tau \varphi+f R \varphi$.

Further calculation in this subsection will essentially repeat the one of [8], but with another potential (53). We include this short review part for making all the presentation more consistent.

We can expand (52) in the Riemann normal coordinates as

$$
\begin{aligned}
-\hat{H}= & \eta^{\mu \nu} \partial_{\mu} \partial_{\nu}+\frac{1}{3} R^{\mu}{ }_{\alpha}{ }^{\nu}{ }_{\beta} y^{\alpha} y^{\beta} \partial_{\mu} \partial_{\nu} \\
& -\frac{2}{3} R^{\alpha}{ }_{\beta} y^{\beta} \partial_{\alpha}+m^{2}-\xi R+V^{\prime \prime}+\cdots .
\end{aligned}
$$

where $\cdots$ denotes high order terms in curvature.

The main advantage of the local momentum representation is that the calculation can be performed in flat space-time and the result can be always presented in a covariant way. For instance, the equation for the propagator of a real scalar field has the form

$\hat{H} G\left(x, x^{\prime}\right)=-\delta^{c}\left(x, x^{\prime}\right)$,

where $\delta^{c}\left(x, x^{\prime}\right)=g^{-\frac{1}{4}}\left(x^{\prime}\right) \delta\left(x, x^{\prime}\right) g^{-\frac{1}{4}}(x)$ is a covariant Dirac delta function.

Since we are going to make calculations around the flat metric, it is most useful to work with the modified propagator $\bar{G}\left(x, x^{\prime}\right)$ where

$\hat{H} \bar{G}\left(x, x^{\prime}\right)=-\delta\left(x, x^{\prime}\right)$

The explicit form of $\bar{G}\left(x, x^{\prime}\right)$ is known $[9,10]$ for the free case when $V^{\prime \prime}=m^{2}$ and for the even potential at constant 
$\varphi[8]$. As far as it is sufficient to regard $V^{\prime \prime}=$ const for the derivation of effective potential, we can replace $m^{2}$ by $\tilde{m}^{2}=V^{\prime \prime}$ and obtain, in first order of curvature expansion, the following expression:

$$
\begin{aligned}
\bar{G}(y)= & \int \frac{d^{4} k}{(2 \pi)^{4}} e^{i k x}\left[\frac{1}{k^{2}-\tilde{m}^{2}}\right. \\
& \left.-\left(\xi-\frac{1}{6}\right) \frac{R}{\left(k^{2}-\tilde{m}^{2}\right)^{2}}\right]+\cdots .
\end{aligned}
$$

We can expand $\hat{H}$ and $\bar{G}\left(x, x^{\prime}\right)$ up to the first power of scalar curvature as

$$
\begin{aligned}
\hat{H} & =\hat{H}_{0}+\hat{H}_{1} R+O\left(R_{\ldots}^{2}\right), \\
\bar{G} & =\bar{G}_{0}+\bar{G}_{1} R+O\left(R_{\ldots}^{2}\right) .
\end{aligned}
$$

Starting from this point the $\mathcal{O}\left(R_{\ldots}^{2}\right)$ terms will not be mentioned.

As far as $\operatorname{Tr} \ln \hat{H}=-\operatorname{Tr} \ln G\left(x, x^{\prime}\right)$, we get

$$
\begin{aligned}
-\frac{1}{2} \operatorname{Tr} \ln \bar{G}\left(x, x^{\prime}\right) & =\frac{1}{2} \operatorname{Tr} \ln \left(\hat{H}_{0}+\hat{H}_{1} R\right) \\
& =\frac{1}{2} \operatorname{Tr} \ln \hat{H}_{0}+\frac{1}{2} \operatorname{Tr}\left(\bar{G}_{0} \hat{H}_{1} R\right) .
\end{aligned}
$$

Consider first the effective potential in flat space. The first term in the r.h.s of (57) includes the flat space contribution $\bar{V}_{0}(\varphi)$, which can be defined as

$\bar{V}_{0}(\varphi)=\frac{1}{2} \operatorname{Tr} \ln S_{2}(\varphi)-\frac{1}{2} \operatorname{Tr} \ln S_{2}(\varphi=0)$.

Here $S_{2}$ is the bilinear form of the classical scalar field action,

$S_{2}(\varphi)=\frac{1}{2} \int d^{4} x\left\{\varphi \eta^{\mu \nu} \partial_{\mu} \partial_{\nu} \varphi+V^{\prime \prime}\right\}$.

As a result we have

$\bar{V}_{0}(\varphi)=\frac{1}{2} \operatorname{Tr} \ln \left\{\frac{\square+V^{\prime \prime}}{\square+m^{2}}\right\}$.

Unlike the previous sections, all the subsequent calculations will be performed in the cut-off regularization what helps to simplify the calculations and make them more explicit. The transition to dimensional regularization can be easily done, of course. By introducing the four-dimensional Euclidean momentum cut-off $\Omega$ and integrating over angular coordinates, we arrive at [8]

$\bar{V}_{0}(\varphi)=\frac{1}{2(4 \pi)^{2}} \int_{0}^{\Omega} k^{2} d k^{2} \ln \left(\frac{k^{2}+V^{\prime \prime}}{k^{2}+m^{2}}\right)$.

Taking the last integral, after some algebra we obtain

$\bar{V}_{0}\left(\varphi, \eta_{\mu \nu}\right)=\bar{V}_{0}^{d i v}+\bar{V}_{0}^{\text {fin }}$,

$V_{0}^{d i v}=\frac{1}{2(4 \pi)^{2}}\left\{\Omega^{2} V^{\prime \prime}-\frac{1}{2}\left(V^{\prime \prime}\right)^{2} \ln \frac{\Omega^{2}}{m^{2}}\right\}$,
$\bar{V}_{0}^{f i n}=\frac{1}{2(4 \pi)^{2}}\left\{\frac{1}{2}\left(V^{\prime \prime}\right)^{2} \ln \left(1+\frac{V^{\prime \prime}}{m^{2}}\right)-\frac{1}{4}\left(V^{\prime \prime}\right)^{2}\right\}$.

In order to cancel divergences, we follow the minimal substraction scheme and introduce an appropriated counterterm in the form

$$
\begin{aligned}
\Delta V_{0}= & \frac{1}{2(4 \pi)^{2}}\left\{-\Omega^{2} V^{\prime \prime}\right. \\
& \left.+\frac{1}{2}\left(V^{\prime \prime}\right)^{2} \ln \frac{\Omega^{2}}{\mu^{2}}+\frac{1}{4}\left(V^{\prime \prime}\right)^{2}\right\},
\end{aligned}
$$

where $\mu$ is the dimensional renormalization parameter. In this way, the quadratic and logarithmic divergences are eliminated and the renormalized effective potential can be written as

$$
\begin{aligned}
V_{e f f}^{r e n}\left(\eta_{\mu \nu}, \varphi\right) & =V+\bar{V}_{0}+\Delta V_{0} \\
& =V+\frac{\left(V^{\prime \prime}\right)^{2}}{64 \pi^{2}} \ln \left(\frac{V^{\prime \prime}}{\mu^{2}}\right) .
\end{aligned}
$$

Let us consider the linear in curvature corrections. The first order contribution is due to the second term in the r.h.s. of Eq. (58). This term can be easily presented in the form

$$
\begin{aligned}
\frac{1}{2} \operatorname{Tr}\left(\bar{G}_{0} \hat{H}_{1} R\right)= & \frac{1}{2} \int d^{4} x \int d^{4} x^{\prime}\left[\bar{G}_{0}^{-1}\left(x, x^{\prime}\right) \bar{G}_{1}\left(x^{\prime}, x\right)\right] R \\
= & \frac{1}{2} \int d^{4} x \int d^{4} x^{\prime} R \int \frac{d^{4} k}{(2 \pi)^{4}} e^{i k\left(x-x^{\prime}\right)} \\
& \times \int \frac{d^{4} p}{(2 \pi)^{4}} e^{i p\left(x^{\prime}-x\right)} \bar{G}_{0}^{-1}(k) \bar{G}_{1}(p) \\
= & \frac{1}{2} \int d^{4} x R \int \frac{d^{4} k}{(2 \pi)^{4}} \bar{G}_{0}^{-1}(k) \bar{G}_{1}(k)
\end{aligned}
$$

and hence

$$
\begin{aligned}
& \frac{1}{2} \operatorname{Tr}\left(\bar{G}_{0} \hat{H}_{1} R\right)= \\
& -\frac{1}{2(4 \pi)^{2}}\left(\xi-\frac{1}{6}\right) \int d^{4} \times R \int_{0}^{\Omega} \frac{k^{2} d k^{2}}{k^{2}+\tilde{m}^{2}} .
\end{aligned}
$$

After taking the last integral, the final result reads

$$
\bar{V}\left(\varphi, g_{\mu \nu}\right)=\bar{V}_{0}+\bar{V}_{1} R
$$

where $\bar{V}_{0}$ is given by Eq. (64) and $\bar{V}_{1}=\bar{V}_{1}^{\text {fin }}+\bar{V}_{1}^{\text {div }}$, where

$$
\begin{aligned}
& \bar{V}_{1}^{\text {div }}=\frac{1}{2(\pi)^{4}}\left(\xi-\frac{1}{6}\right)\left[-\Omega^{2}+\left(V^{\prime \prime}\right) \ln \frac{\Omega^{2}}{m^{2}}\right], \\
& \bar{V}_{1}^{f i n}=\frac{1}{2(\pi)^{4}}\left(\xi-\frac{1}{6}\right)\left[\left(V^{\prime \prime}\right) \ln \left(\frac{V^{\prime \prime}}{m^{2}}\right)\right] .
\end{aligned}
$$


Similar to the flat space case, the potential must be modified by adding a counterterm,

$$
\Delta \bar{V}_{1}=\frac{1}{2(\pi)^{4}}\left(\xi-\frac{1}{6}\right)\left[\Omega^{2}-\left(V^{\prime \prime}\right) \ln \frac{\Omega^{2}}{\mu^{2}}\right] .
$$

Thus, the renormalized expression is

$$
\begin{aligned}
V_{e f f, 1}^{r e n}\left(g_{\mu \nu}, \varphi\right)= & \frac{1}{2}\left(m^{2}-\xi R\right) \varphi^{2} \\
& -\frac{1}{2(\pi)^{4}}\left(\xi-\frac{1}{6}\right)\left(V^{\prime \prime}\right) \ln \left(\frac{V^{\prime \prime}}{\mu^{2}}\right) .
\end{aligned}
$$

The full renormalized effective potential for the scalar sector of (1) is the sum of expressions (64) and (70),

$$
\begin{aligned}
V_{e f f}^{r e n}\left(g_{\mu \nu}, \varphi\right)= & \rho_{\Lambda}+V+\frac{\hbar}{2(4 \pi)^{2}}\left[\frac{1}{2}\left(V^{\prime \prime}\right)^{2}\right. \\
& \left.-\left(\xi-\frac{1}{6}\right) R\left(V^{\prime \prime}\right)\right] \ln \left(\frac{V^{\prime \prime}}{\mu^{2}}\right),
\end{aligned}
$$

where we restored the first power of the loop parameter $\hbar$ and added the cosmological constant term, $\rho_{\Lambda}$.

\subsection{Fermion contributions and overall expression}

Let us consider now the fermion contribution to the effective potential of the sterile scalar. In the case of potential the background field $\varphi$ can be treated as a constant, hence we denote $\tilde{M}=M+h \varphi$. Taking the Grassmann parity of the quantum field into account, in the Euclidean notations we get

$\Gamma_{f}^{(1)}\left[\varphi, g_{\mu \nu}\right]=-\operatorname{Tr} \ln \hat{H}_{f}$,

where $\hat{H}_{f}=i\left(\gamma^{\mu} \nabla_{\mu}+i \tilde{M}\right) \delta^{i j}$. As usual, we consider [23]

$\operatorname{Tr} \ln \hat{H}_{f}=\frac{1}{2} \operatorname{Tr} \ln \left(\hat{H}_{f} \hat{H}_{f}^{*}\right)$,

with $\hat{H}_{f}^{*}=i\left(\gamma^{\mu} \nabla_{\mu}-i \tilde{M}\right) \delta_{j k}$. After some algebra this gives

$\operatorname{Tr} \ln \hat{H}_{f}=\frac{1}{2} \operatorname{Tr} \ln \left(-\square+\frac{1}{4} R-\tilde{M}^{2}\right) \delta_{k}^{i}$

The fermion propagator is defined from the relation

$\left(\hat{H}_{f} \hat{H}_{f}^{*}\right) \mathcal{G}\left(x, x^{\prime}\right)=-\delta^{c}\left(x, x^{\prime}\right)$.

Following the same scheme which was used in the scalar case, one can define modified propagator

$\overline{\mathcal{G}}\left(x, x^{\prime}\right)=\overline{\mathcal{G}}\left(x, x^{\prime}\right) g^{-\frac{1}{4}}(x)$,

which satisfies the equation

$\left(\hat{H}_{f} \hat{H}_{f}^{*}\right) \overline{\mathcal{G}}\left(x, x^{\prime}\right)=-\delta\left(x, x^{\prime}\right)$.
From the paper by Bunch and Parker [9] we learn that $\overline{\mathcal{G}}\left(x, x^{\prime}\right)=\overline{\mathcal{G}}(y)$ is defined as

$\overline{\mathcal{G}}(y)=\int \frac{d^{4} k}{(2 \pi)^{4}} e^{i k y}\left[1-\frac{1}{12} R \frac{\partial}{\partial \tilde{M}^{2}}\right]\left(k^{2}+\tilde{M}^{2}\right)^{-1} \hat{1}$

in the first order in curvature. Thus,

$-\frac{1}{2} \operatorname{Tr} \ln \mathcal{G}\left(x, x^{\prime}\right)=\frac{1}{2} \operatorname{Tr} \ln \hat{H}_{f}=\frac{1}{4} \operatorname{Tr} \ln \left(\hat{H}_{f} \hat{H}_{f}^{*}\right)$.

Using the same considerations as for the scalar field, we find that

$$
\begin{aligned}
-\frac{1}{2} \operatorname{Tr} \ln \mathcal{G}\left(x, x^{\prime}\right)= & \frac{1}{4} \operatorname{Tr} \ln \left(\hat{H}_{f} \hat{H}_{f}^{*}\right)_{0} \\
& +\frac{1}{4} \operatorname{Tr} \overline{\mathcal{G}}_{0}\left(\hat{H}_{f} \hat{H}_{f}^{*}\right)_{1} R
\end{aligned}
$$

The first term in r.h.s. correspond to the flat space case and the second one is the first order in curvature contribution. We will first perform the calculation in the flat space, when

$\frac{1}{4} \operatorname{Tr} \ln \left(\hat{H}_{f} \hat{H}_{f}^{*}\right)_{0}=\frac{1}{4} \operatorname{sTr} \ln \left(-\eta^{\mu v} \partial_{\mu} \partial_{v}-\tilde{M}^{2}\right) \delta_{k}^{i}$.

In the momentum representation this gives

$\frac{1}{4} \operatorname{Tr} \ln \left(\hat{H}_{f} \hat{H}_{f}^{*}\right)_{0}=(-2 N) \int_{0}^{\Omega} \frac{d k^{2}}{(4 \pi)^{2}} k^{2} \ln \left(\frac{1}{k^{2}+\tilde{M}^{2}}\right)$,

which provides the flat space part of the one-loop effective potential,

$V_{0}^{d i v}($ fer $)=-\frac{2 N}{(4 \pi)^{2}}\left\{\frac{1}{2} \ln \left(\frac{\Omega^{2}}{\tilde{M}^{2}}\right) \tilde{M}^{4}+\frac{1}{4} \Omega^{2}\right\}$.

In order to renormalize this result, we introduce a counterterm of the form

$\Delta V_{0}=\frac{2 N}{(4 \pi)^{2}}\left\{\frac{1}{2} \ln \left(\frac{\Omega^{2}}{\mu^{2}}\right) \tilde{M}^{4}+\frac{1}{4} \Omega^{2}\right\}$

Thus,

$V_{0}^{r e n}(f e r)=\frac{N}{(4 \pi)^{2}} \ln \left(\frac{\tilde{M}^{2}}{\mu^{2}}\right) \tilde{M}^{4}$

The contribution in first order of curvature is

$$
\begin{aligned}
& \frac{1}{4} \operatorname{Tr} \overline{\mathcal{G}}_{0}\left(\hat{H}_{f} \hat{H}_{f}^{*}\right)_{1} R \\
& \quad=(-2 N) \int d^{4} x R \times \int \frac{d^{4} k}{(2 \pi)^{4}} \overline{\mathcal{G}}_{0}^{-1}(k) \overline{\mathcal{G}}_{1}(k) .
\end{aligned}
$$

which can be written in momentum space as

$$
\begin{aligned}
& \frac{1}{4} \operatorname{Tr} \overline{\mathcal{G}}_{0}\left(\hat{H}_{f} \hat{H}_{f}^{*}\right)_{1} R \\
& \quad=-\frac{N}{6(4 \pi)^{2}} R \int_{0}^{\Omega} d k^{2} \frac{k^{2}}{k^{2}+\tilde{M}^{2}} .
\end{aligned}
$$


Therefore,

$V_{1}^{d i v}(f e r) R=\frac{N}{6(4 \pi)^{2}} R\left\{\tilde{M}^{2} \ln \frac{\Omega^{2}}{\tilde{M}^{2}}+\Omega^{2}\right\}$,

while there is no remnant finite part in this case. The divergences can be eliminated by adding a counterterm

$\Delta V_{1}=-\frac{N}{6(4 \pi)^{2}}\left\{\tilde{M}^{2} \ln \frac{\Omega^{2}}{\mu^{2}}+\Omega^{2}\right\}$.

Finally, the first order in curvature part of the renormalized effective potential has the form

$V_{\text {ren }}($ fer $)=-\frac{N}{(4 \pi)^{2}}\left\{\tilde{M}^{4}-\frac{1}{6} R \tilde{M}^{2}\right\} \ln \left(\frac{\tilde{M}^{2}}{\mu^{2}}\right)$.

Summing up the scalar (71) and fermion (87) contributions, we arrive at the general expression for the effective potential of our model, which includes a single real sterile scalar and $N$ copies of massive fermion fields,

$$
\begin{aligned}
V_{e f f}^{r e n}\left(g_{\mu \nu}, \varphi\right)= & \rho_{\Lambda}+\frac{1}{2}\left(m^{2}-\xi R\right) \varphi^{2}+V \\
& +\frac{\hbar}{2(4 \pi)^{2}}\left\{\left[\frac{1}{2}\left(V^{\prime \prime}+m^{2}\right)^{2}\right.\right. \\
& \left.-\left(\xi-\frac{1}{6}\right) R\left(V^{\prime \prime}+m^{2}\right)\right] \ln \left(\frac{V^{\prime \prime}+m^{2}}{\mu^{2}}\right) \\
& -2 N(M+h \varphi)^{4} \ln \left[\frac{(M+h \varphi)^{2}}{\mu^{2}}\right] \\
& \left.+\frac{N}{3} R(M+h \varphi)^{2} \ln \left[\frac{(M+h \varphi)^{2}}{\mu^{2}}\right]\right\},(88)
\end{aligned}
$$

where the interacting and odd terms of the classical potential $V$ (remember that we separated the term with scalar mass for the sake of convenience) are defined in Eq. (53). It is easy to verify the perfect correspondence with the expression (50) derived from the Minimal Subtraction-based renormalization group with the scale identifications (35) and (46).

The effective potential (88) depends on an arbitrary parameter $\mu$. To fix the value of this parameter, we should imposed the renormalization conditions in a usual way.

\section{Induced action of gravity with odd terms}

In this section we discuss some interesting aspects of the model under consideration related to symmetry breaking, induced action of gravity and its possible physical manifestations.

In the scalar theory in flat space without odd terms in the scalar sector, the classical potential $U=-\frac{1}{2} m^{2} \varphi^{2}+V$ with "untrue" sign at $m^{2}$ has a constant minimum position, which corresponds to the spontaneous symmetry breaking (SSB) of discrete symmetry. In curved space such a constant position of the minimum and corresponding vacuum state are impossible [21]. The most immediate reason is that the classical potential contains the non-minimal $\xi R(x) \varphi^{2}$ term and constant solution for the scalar field is impossible in the general case of an arbitrary metric. Such a general analysis is beyond the scope of this paper, the discussion of related issues can be found e.g. in Ref. [22].

Things get even more complicated in the case of the sterile scalar, since the odd powers of the scalar in the potential make the discrete symmetry impossible and hence there is no much sense to speak about its breaking. However, let us assume that odd terms and also the more traditional non-minimal term $\xi R \varphi^{2}$ are small and treat them as small perturbations. In this case we have a SSB in the zero-order approximation, and this is the terminology which we shall adapt in what follows.

Let us consider the equation of motion for vacuum expectation value (VEV) of the scalar field.

$\left.\frac{\delta S}{\delta \varphi}\right|_{\varphi=v}=0$

where $v$ is supposed to be constant and in many cases it is so. However, as we have just mentioned, the solution of this problem in curved space is quite nontrivial [21], because in general $R$ is not constant and $\xi \neq 0$. As a result, the equation for the VEV

$-\square v+m^{2} v+\xi R v-\frac{1}{6} \lambda v^{3}-\frac{1}{2} g v^{2}-\tau-f R=0$

can not be solved with constant $v$ even neglecting the kinetic term. Following [21] we can expand the solution into power series in the curvature tensor or $\xi$, such that

$v(x)=v_{0}+v_{1}(x)+v_{2}(x)+\cdots$.

In the solution of the problem of VEV we shall treat both $\xi R$ and odd terms as small perturbations.

In the zero order we have

$v_{0}^{2}=\frac{6 m^{2}}{\lambda}$

Now, in order to solve Eq. (90) in the first order, consider the following approximations:

$|g| \ll v_{0}, \quad|\tau| \ll v_{0}^{3}, \quad|\xi R| \ll v_{0}^{2}, \quad|f| \ll v_{0}$. 
After solving the perturbative problem independently for $\xi R$ and odd terms, and summing up the results we obtain

$v(x)=v_{0}-\frac{\tau}{\mu_{0}^{2}+g v_{0}}+\frac{\xi v_{0}-f}{\square+\mu_{0}^{2}} R$,

where

$\mu_{0}^{2}=\frac{\lambda}{3} v_{0}^{2}$.

To obtain the induced low-energy action we substitute the solution (94) into the action (1). The result has the form

$$
\begin{aligned}
S_{\text {ind }}= & \int d^{4} x \sqrt{-g}\left\{-\rho_{\Lambda}^{\text {ind }}-\frac{1}{16 \pi G_{\text {ind }}} R\right. \\
& +\left(\tau f-\tau \xi v_{0}\right) \frac{1}{\square+\mu_{0}^{2}} R+\left(f \xi v_{0}-\xi^{2} v_{0}^{2}\right. \\
& \left.\left.-\frac{1}{3} g \xi v_{0}^{3}+\frac{1}{3} g f v_{0}^{2}-f \xi v_{0}+f^{2}\right) R \frac{1}{\square+\mu_{0}^{2}} R\right\} .
\end{aligned}
$$

In the expression (96) the cosmological constant and the inverse Newton constant are defined by the expressions

$$
\begin{aligned}
\rho_{\Lambda}^{i n d}= & -\frac{\lambda}{24} v_{0}^{4}-\frac{4}{3} \frac{\tau \lambda v_{0}^{3}}{\mu_{0}^{2}+g v_{0}}+\frac{1}{6} g v_{0}^{3} \\
& +\tau v_{0}-\frac{\tau^{2}}{\mu_{0}^{2}+g v_{0}}, \\
\frac{1}{16 \pi G_{\text {ind }}}= & \frac{\tau v_{0} \xi}{\mu_{0}^{2}+g v_{0}}-\frac{\xi v_{0}}{2}+f v_{0}-\frac{\tau f}{\mu_{0}^{2}+g v_{0}} .
\end{aligned}
$$

As expected, these formulas show the small contributions of the odd parameters of the sterile scalar $\tau, f$ and $g$. Since these parameters are certainly small, the change in these induced quantities is irrelevant compared to the quantities induced, e.g., in the electroweak phase transition, where $v_{0}$ is the vacuum expectation value of the Higgs field and the mentioned induced quantities may be much larger that, for instance, for the quintessence field.

At the same time the induced action (96) have two other details which may be in fact more significant. First of all, in the case of quintessence the mass scale should be very small, and hence the $\mathrm{VeV}$ value $v_{0}$ should be small too. Then the non-local terms in the second line of Eq. (96) may become phenomenologically relevant. It would be interesting to explore the phenomenological limits on the odd parameters, starting from $v_{0}$ from the experimental data on Newton law and observational data on the bending of light, and see whether these limits can produce some restrictions on the quintessence potential. This investigation is beyond the scope of the present work, since it is devoted to the quantum aspects of a sterile scalar, however even the possibility looks attractive.

Another aspect concerns the term $\left(\square+\mu_{0}^{2}\right)^{-1} R$ in the first line of Eq. (96). According to the recent discussion in Refs. $[24,25]$ this term can be relevant in cosmology, in the periods when the inverse of the size of the horizon may be comparable with the cosmic scale. Up to some extent, the corresponding effect can lead to the change of observational predictions in both inflationary and late cosmology epochs.

Let us now consider an application of odd terms in the scalar action (1) to inflation. Since the present-day reference theory for inflation is the one of Starobinsky [26], the simplest approach is to make a mapping to the $R+F(R)$ and take care about the effect of the odd terms. As usual, we consider that the non-minimal term and the $\lambda \varphi^{4}$-terms in the classical potential (41) are dominating, while the mass $m^{2} \varphi^{2}$-term, classical odd terms and the leading quantum corrections in the effective potential (50) are small and can be regarded as perturbations. As a first approximations and for the sake of simplicity, the kinetic $(\partial \varphi)^{2}$-term can be completely omitted, assuming that $R$ is almost constant in the inflationary period. Let us stress that the detailed analysis of inflation in the model under consideration is beyond the scope of the present work, we are just trying to sketch the main features of the inflationary model with odd terms, and leave the rest of the work for the future.

The derivation of the induced action of gravity in this approximation differs from what we have done above. The equation for the scalar field is

$\xi R \varphi-\frac{1}{6} \lambda \varphi^{3}-m^{2} \varphi-\frac{1}{2} g \varphi^{2}-\tau-f R=0$.

In the zero-order approximation we get

$\xi R \varphi_{0}-\frac{1}{6} \lambda \varphi_{0}^{3}=0 \Longrightarrow \varphi_{0}^{2}=\frac{4 \xi R}{\lambda}$.

Let us remark that since during inflation $R$ is negative and we need a positive $\lambda$ for the stability of the potential, this solution implies $\xi<0$, such that the product $\xi R>0$. Substituting (100) back into the action, in the leading order there is an induced term $\frac{3 \xi^{2}}{2 \lambda} R^{2}$. According to the classical estimate of [27], this means that the ratio $\frac{3 \xi^{2}}{2 \lambda}$ should be close to $5 \times 10^{8}$ to provide a successful model of inflation.

In the next approximation we consider $\varphi=\varphi_{0}+\varphi_{1}$, and arrive at the linear equation for $\varphi_{1}$,

$$
\left(\xi R-\frac{\lambda}{2} \varphi_{0}^{2}\right) \varphi_{1}=m^{2} \varphi_{0}+\frac{g}{2} \varphi_{0}^{2}+\tau+f R,
$$


which solves in the form

$\varphi_{1}=-m^{2} \sqrt{\frac{3}{2 \lambda \xi R}}-\frac{3 g}{2 \lambda}-\frac{\tau}{2 \xi R}-\frac{f}{2 \xi}$.

After placing the sum $\varphi=\varphi_{0}+\varphi_{1}$ into the action, in the leading order we get the induced Lagrangian of gravity that corresponds to the given approximation,

$$
\begin{aligned}
L_{\text {ind }}= & -\frac{3 m^{2} \xi}{\lambda} R+\frac{3 \xi^{2}}{2 \lambda} R^{2} \\
& +\sqrt{\frac{6 \xi R}{\lambda}}\left[\tau-\left(f+\frac{g \xi}{\lambda}\right) R\right] .
\end{aligned}
$$

The first term in the this expression is a small irrelevant addition to the classical Einstein-Hilbert term $\sim M_{P}^{2} R$. The second term in the first line is the leading $R^{2}$-term which was mentioned above, it represents the main element of the Starobinsky inflation [26,27]. According to our approximation, the terms in the second line of (103) represent a small addition to the main $R^{2}$-term. The analysis of the effect of these terms on the inflationary observables (mainly the spectral index $n_{s}$ of the primordial curvature perturbations and the tensor-to-scalar ratio $r$ ) can be done along the way of the previous works in this direction [28,29].

The comparison to the polynomial model of perturbations in [28] is especially instructive. Let us stress that the odd terms in the classical potential of the sterile scalar field are necessary to provide a quantum consistency of the theory of a sterile scalar coupled to fermions. Our consideration shows that this implies the induced gravitational action to include the non-polynomial terms (103). As we mentioned above, the detailed analysis of these terms is beyond the scope of the present work. However, we can use the renormalization group equations (26) for the parameters of the action, together with the estimate [27] (see also recent paper [30]) for the ratio $\frac{3 \xi^{2}}{2 \lambda} \propto 5 \times 10^{8}$, to evaluate the magnitudes of the induced non-polynomial terms.

Assuming that $\lambda \approx 1$, we arrive at the estimate $|\xi| \approx$ $4 \times 10^{4}$, similar to the Higgs inflation [31]. Next, according to the equations (26), the lower bound for the absolute values of the odd parameters is defined by the mass of the heaviest fermion that couples to the sterile scalar, multiplied by the corresponding Yukawa coupling. Assuming that this coupling is of the order one (which can be, in principle, justified by the need to create fermionic particles from the vacuum after inflations), we get an estimate

$g \sim m_{f}, \quad \tau \sim m_{f}^{3}, \quad f \sim m_{f}$.

For the heaviest particle of the Minimal Standard Model, the top-quark, we have $m_{f}=m_{t}=175 \mathrm{GeV}$. On the top of that we can use the value of Hubble parameter during inflation,

$H_{\text {inf }}=10^{11}-10^{13} \mathrm{GeV}$, with $|R| \sim H_{\text {inf }}^{2}$,

and this gives the estimates

$\sqrt{\frac{6 \xi R}{\lambda}} \sim 10^{20} \mathrm{GeV}$.

It is easy to see that our "optimistic" estimates lead to the situation that the magnitudes of the non-polynomial terms in the second line of (103) are about 3-4 orders of magnitude smaller that the main $R^{2}$-term in the first line. The important consequences of this fact are that (i) the most relevant odd parameter is $g$, since its contribution is many orders of magnitude greater that the ones of of $\tau$ and even $f$. (ii) the treatment of the non-polynomial in $R$ terms as small additions to the main even part, and consequently the same treatment for the odd terms in the potential compared to the even terms, is justified. (iii) the interaction of the inflaton with much heavier fermions, such as the ones of supersymmetric GUT's may lead to the real trouble with the odd terms in inflation. In this case the non-polynomial terms become dominating and this implies the conflict with the observational data, e.g. with the ones of Planck [32]. This problem can be of course solved by imposing the small Yukawa couplings for these fermions, that can be seen as a general restriction for the inflaton-based models. It is interesting that we arrived at this conclusion just by requiring the consistency of the theory of inflaton at the quantum level.

\section{Conclusions and perspectives}

As we have seen in the previous sections, in the model with sterile scalar interacting to fermions there is no symmetry protection from the terms which are odd in the field, as a result these odd terms are necessary for the renormalizability of the theory. Similar conclusion has been done recently in $[3,4]$, but we were trying to make renormalization in a consistent way that requires including odd terms into the classical potential.

As far as we include these terms into the classical action, the induced action of gravity, including both cosmological and inverse Newton constants, starts to depend on the new terms. And the most dramatic effect is that the induced action (4) gains the non-local contributions with the very small mass (3) in the Green function. This illustrated the effect which the sterile scalar (such as, e.g., quintessence) can produce on the gravitational action. It would be interesting to explore the effect of these non-local terms at the phenomenological level. 
An interesting consequence can be met by requiring the quantum consistency of the inflaton coupled to fermions. The simple mapping to the $F(R)$ models in (103) shows that the odd terms in the scalar potential produce the non-polynomial terms in the gravitational action, with several relevant consequences. In particular, there may be essential restrictions on the Yukawa interaction of the inflaton to the heavy fermions beyond the Standard Model, coming from the Planck data [32].

Acknowledgements The work of I.Sh. was partially supported by Conselho Nacional de Desenvolvimento Científico e Tecnológico CNPq, 303893/2014-1 and Fundação de Amparo à Pesquisa de Minas Gerais - FAPEMIG, APQ-01205-16. A.J.V. are grateful to Coordenação de Aperfeiçoamento de Pessoal de Nível Superior - CAPES for supporting their Ph.D. and MSc projects. I.B. is grateful to CAPES for supporting his long-term visit to UFJF and to the Physics Departament of UFJF for kind hospitality. Also he thanks the Russian Ministry of Science and High Education, project No. 3.1386.2017 for partial support.

Data Availability Statement This manuscript has associated data in a data repository. [Authors' comment: The datasets generated during and/or analysed during the current study are available from the corresponding author on reasonable request.]

Open Access This article is distributed under the terms of the Creative Commons Attribution 4.0 International License (http://creativecomm ons.org/licenses/by/4.0/), which permits unrestricted use, distribution, and reproduction in any medium, provided you give appropriate credit to the original author(s) and the source, provide a link to the Creative Commons license, and indicate if changes were made.

Funded by SCOAP ${ }^{3}$.

\section{Appendix}

The intermediate expressions leading to (13) are

$$
\begin{aligned}
& \nabla_{\mu} \hat{h}^{\mu}=\left(\begin{array}{cc}
0 & \frac{i}{2} h \nabla_{\mu} \bar{\Psi}_{j} \gamma^{\mu} \\
0 & \frac{i}{2} h \nabla_{\mu} \varphi \gamma^{\mu} \delta^{i j}
\end{array}\right), \hat{h}_{\mu} \hat{h}^{\mu}=\left(\begin{array}{c}
0-h^{2} \bar{\Psi}_{k} \varphi \\
0-h^{2} \varphi^{2} \delta^{i k}
\end{array}\right) \\
& \text { and } \hat{h}_{\mu} \hat{h}_{\nu}=\left(\begin{array}{c}
0-\frac{1}{4} h^{2} \bar{\Psi}_{k} \varphi \gamma_{\mu} \gamma_{\nu} \\
0-\frac{1}{4} h^{2} \varphi^{2} \gamma_{\mu} \gamma_{\nu} \delta^{i k}
\end{array}\right) .
\end{aligned}
$$

Thus, we arrive at the expressions

\section{References}

1. I.L. Buchbinder, I.L. Shapiro, On the renormalization group equations in curved space-time with torsion. Class. Quant. Grav. 7, 1197 (1990)

2. I.L. Buchbinder, S.D. Odintsov, I.L. Shapiro, Effective Action in Quantum Gravity (IOP Publishing, Bristol, 1992)

3. D.J. Toms, Effective action for the Yukawa model in curved spacetime. JHEP 1805, 139 (2018). arXiv: 1804.08350

4. D.J. Toms, Gauged Yukawa model in curved spacetime. Phys. Rev. D 98, 025015 (2018). arXiv: 1805.01700

5. I.L. Buchbinder, S.D. Odintsov, Effective Potential In A Curved Space-time. Sov. Phys. J. 27, 554 (1984)

6. I.L. Buchbinder, J.J. Wolfengaut, Renormalization group equations and effective action in curved space-time. Class. Quant. Grav. 5, 1127 (1988)

7. S.R. Coleman, E.J. Weinberg, Radiative corrections as the origin of spontaneous symmetry breaking. Phys. Rev. D 7, 1888 (1973)

8. F. Sobreira, B.J. Ribeiro, I.L. Shapiro, Effective potential in curved space and cut-off regularizations. Phys. Lett. B 705, 273 (2011). arXiv: 1107.2262

9. T.S. Bunch, L. Parker, Feynman propagator in curved space-time: a momentum space representation. Phys. Rev. D 20, 2499 (1979)

10. L. Parker, D.J. Toms, Renormalization group analysis of grand unified theories in curved space-time. Phys. Rev. D 29, 1584 (1984)

11. I.L. Buchbinder, I.L. Shapiro, Gravitational interaction effect on behavior of the yukawa and scalar effective coupling constants. Yad. Fiz. 44 , 1033 (1986) (in Russian)

12. I.L. Buchbinder, O.K. Kalashnikov, I.L. Shapiro, V.B. Vologodsky, J.J. Wolfengaut, The stability of asymptotic freedom in grand unified models coupled to $R^{2}$ gravity. Phys. Lett. B 216, 127 (1989)

13. I.L. Shapiro, Asymptotical behavior of effective Yukawa coupling constants in quantum $R^{2}$-gravity. Class. Quant. Grav. 6, 1197 (1989)

14. A. Salvio, A. Strumia, Agravity. JHEP 1406, 080 (2014). arXiv: 1403.4226

15. N.D. Birell, P.C.W. Davies, Quantum Fields in Curved Space (Cambridge University Press, Cambridge, 1982)

16. A.O. Barvinsky, G.A. Vilovisky, The generalized SchwingerDewitt technique in gauge theories and quantum gravity. Phys. Repts. 119, 1 (1985)

17. G. de Berredo-Peixoto, D.D. Pereira, I.L. Shapiro, Universality and ambiguity in fermionic effective actions. Phys. Rev. D D85, 064025 (2012). arXiv:1201.2649

18. G. de Berredo-Peixoto, E.V. Gorbar, I.L. Shapiro, On the renormalization group for the interacting massive scalar field theory in curved space. Class. Quant. Grav. 21, 2281 (2004). arxiv:hep-th/0311229

19. I.L. Buchbinder, On renormalization group equations in curved space-time. Theoret. Math. Phys. 61, 1215 (1984) (Teoret.mat.fiz. 61 (1984) 393)

$$
\begin{gathered}
\hat{P}=\left(\begin{array}{rr}
\frac{\lambda \varphi^{2}}{2}+g \varphi+m^{2}-\left(\xi-\frac{1}{6}\right) R & h M \bar{\Psi}_{k}-\frac{i}{2} h\left(\nabla_{\mu} \bar{\Psi}_{k}\right) \gamma^{\mu}+h^{2} \bar{\Psi}_{k} \varphi \\
2 h \Psi_{i} & {\left[M^{2}-\frac{1}{12} R+h M \varphi-\frac{i}{2} h\left(\nabla_{\mu} \varphi\right) \gamma^{\mu}+h^{2} \varphi^{2}\right] \delta^{i k}}
\end{array}\right) \\
\text { and } \left.\quad \hat{S}_{\mu \nu}=\left(\begin{array}{l}
0 \\
0\left[\frac{1}{4} R_{\mu \nu \alpha \beta} \gamma^{\alpha} \gamma^{\beta}-\frac{i}{2} h\left(\left(\nabla_{\mu} \varphi\right) \gamma_{\nu}-\left(\nabla_{\nu} \varphi\right) \gamma_{\mu}\right)+\frac{1}{4} h^{2} \varphi^{2}\left[\gamma_{\mu}, \gamma_{\nu}\right]\right.
\end{array}\right] \delta^{i k}\right)
\end{gathered}
$$


20. D.J. Toms, The effective action and the renormalization group equation in curved space-time. Phys. Lett. B 126, 37 (1983)

21. E.V. Gorbar and I.L. Shapiro, renormalization group and decoupling in curved space: III. The case of spontaneous symmetry breaking. JHEP 02, 060 (2004). arxiv:hep-ph/0311190

22. M. Asorey, P.M. Lavrov, B.J. Ribeiro, I.L. Shapiro, Vacuum stress-tensor in SSB theories. Phys. Rev. D 85, 104001 (2012). arXiv: 1202.4235

23. G. De Berredo-Peixoto, A Note on the heat kernel method applied to fermions. Mod. Phys. Lett. A 16, 2463 (2001). hep-th/0108223

24. S.A. Franchino-Viñas, T. de Paula Netto, I.L. Shapiro, O. Zanusso, Form factors and decoupling of matter fields in four-dimensional gravity. Phys. Lett. B 790, 229 (2019). arXiv:1812.00460

25. S. A. Franchino-Viñas, T. de Paula Netto, O. Zanusso, Vacuum effective actions and mass-dependent renormalization in curved space. arXiv: 1902.03167

26. A.A. Starobinski, A new type of isotropic cosmological models without singularity. Phys. Lett. B 91, 99 (1980)
27. A.A. Starobinsky, The perturbation spectrum evolving from a nonsingular initially de-Sitter cosmology and the microwave background anisotropy. Sov. Astron. Lett. 9, 302 (1983)

28. Q.G. Huang, A polynomial $\mathrm{f}(\mathrm{R})$ inflation model. JCAP 1402, 035 (2014). arXiv:1309.3514

29. A.R.R. Castellanos, F. Sobreira, I.L. Shapiro, A.A. Starobinsky, On higher derivative corrections to the $R+R^{2}$ inflationary model. JCAP 1812, 007 (2018). arXiv: 1810.07787

30. T.d.P. Netto, A.M. Pelinson, I.L. Shapiro and A.A. Starobinsky, From stable to unstable anomaly-induced inflation. Eur. Phys. J. C 76, 544 (2016). arXiv:1509.08882

31. F.L. Bezrukov, M. Shaposhnikov, The Standard Model Higgs boson as the inflaton. Phys. Lett. B 659, 703 (2008). arXiv:0710.3755

32. P. A. R. Ade et al. [Planck Collaboration], Planck 2015 results. XX. Constraints on inflation, Astron. Astrophys. 594, A20 (2016). arXiv: 1502.02114 